\title{
El hospital como centro nuclear para mejorar la enseñanza de la medicina
}

\author{
Fernando Civeira Murillo
}

\section{Definición}

El Real Decreto 1558/1986, de 28 de junio, por el que se establecen las bases generales del régimen de conciertos entre las universidades y las instituciones sanitarias, define de forma genérica al hospital universitario como aquel que:

- Posee la infraestructura necesaria para el desarrollo de las funciones docentes e investigadoras.

- Cumple necesidades docentes e investigadoras de una universidad.

- Es incluido en su integridad o a la mayoría de sus servicios en el correspondiente concierto entre la universidad y la institución sanitaria.

En el mismo decreto se intenta concretar las infraestructuras necesarias de la siguiente forma:

- Adscripción a un área de salud (instituciones de titularidad pública).

- Estructura y organización compatible con la docencia e investigación universitaria.

- La integración de al menos tres centros de atención primaria.

- Un conjunto suficiente de servicios y/o unidades asistenciales.

- Un número y variedad suficientes de patologías.

- Protocolos de actuación asistencial.

- Métodos de control y evaluación de la calidad asistencial.

- Infraestructura suficiente para el desarrollo de la investigación clínica y científica.

Es decir, la única definición actual de hospital universitario es muy genérica y se refiere a hos- pitales generales con una organización e infraestructuras compatibles con la docencia e investigación universitaria.

\section{El hospital en la docencia de la medicina}

Resulta incuestionable que la docencia práctica junto al enfermo es fundamental en la formación de los estudiantes de medicina, y que todas las recomendaciones docentes actuales hacen una valoración creciente de la importancia de habilidades y actitudes prácticas de los estudiantes. Sin embargo, la asistencia sanitaria actual se caracteriza por:

- Una gran diversificación asistencial hospitalaria y extrahospitalaria, donde el hospital es solamente una parte, a veces poco relevante, del proceso asistencial que se desarrolla en ámbitos cada vez más heterogéneos: atención primaria, consultas de enfermería, servicios concertados, hospitalización a domicilio, paliativos, servicios de urgencias...

- Predominio de enfermedades crónicas donde el hospital de agudos ve una parte muy parcial del proceso.

- Valor creciente de medidas preventivas que en su mayor parte se realizan fuera del ambiente hospitalario.

- Estancias hospitalarias muy cortas, muy tecnificadas, que suponen un periodo corto del proceso y poco relevante para el estudiante.

- Complejidad de la organización hospitalaria, que depende de un poder político con una preocupación básicamente asistencial.
Catedrático de Medicina. Vicedecano de Hospitales. Hospital Universitario Miguel Servet. Universidad de Zaragoza.

E-mail

civeira@unizar.es 


\section{¿Necesita la universidad de hospitales universitarios para la docencia del pregrado?}

La respuesta es compleja. En mi opinión, para la docencia del pregrado son necesarios hospitales generales (no necesariamente universitarios), pero también centros de salud, servicios de urgencia extrahospitalarios, hospitales geriátricos, consultas de especialidades, unidades de paliativos y un largo etcétera que no implica que deban tener el calificativo de 'universitario'. Por tanto, la universidad precisa una mayor flexibilidad para poder concertar múltiples servicios asistenciales para la docencia del pregrado, y entonces la figura del hospital universitario no es imprescindible para esta función.

\section{Si no son imprescindibles para la docencia pregraduada, ¿necesita la universidad de hospitales universitarios?}

Siendo importante la docencia del pregrado, la universidad realiza un servicio público muy superior. Son funciones de la universidad al servicio de la sociedad:

- La creación, desarrollo, transmisión y crítica de la ciencia, de la técnica y de la cultura.

- La preparación para el ejercicio de actividades profesionales que exijan la aplicación de conocimientos y métodos científicos y para la creación artística.

- La difusión, la valorización y la transferencia del conocimiento al servicio de la cultura, de la calidad de la vida y del desarrollo económico.

- La difusión del conocimiento y la cultura a través de la extensión universitaria y la formación a lo largo de toda la vida.

Para todas estas funciones, el hospital universitario resulta imprescindible y en todos los países avanzados el hospital universitario es el centro de la actividad universitaria en el ámbito biomédico.

\section{Visión del hospital universitario}

El hospital universitario debe definirse como 'una unidad académico-asistencial que integra en todo su funcionamiento el máximo nivel de desarrollo de sus tres misiones: asistencial, docente e investigadora. Altamente reconocido por la sociedad por el excelente nivel de servicio que proporciona, por la alta cualificación de los profesionales que forma, y por ser un lugar que favorece la creación, transmisión y crítica de las ciencias biomédicas, y el desarrollo científico y personal de sus profesionales'.

\section{Análisis de situación de los hospitales universitarios en España}

A pesar de la importante función social universitaria de los hospitales universitarios, éstos presentan diferentes problemas:

- Los conciertos en universidades e instituciones sanitarias están fundamentados en docencia de pregrado y en su mayor parte no contemplan otras aspectos universitarios sustantivos con la universidad.

- Esta docencia de pregrado es un tema marginal para el hospital y, por tanto, no afecta a su funcionamiento.

- El modelo organizativo del supuesto 'hospital universitario' no precisa ser modificado por el concierto. Un hospital pasa a ser universitario 'por decreto', sin existir cambios estructurales en él.

- La función de los profesores de universidad en los hospitales universitarios no está bien definida y en la práctica su trabajo resulta un pluriempleo: unas horas para la universidad y unas horas para el hospital. Esto genera una enorme insatisfacción para la mayor parte de los profesionales y conflictos permanentes entre instituciones.

- La docencia del pregrado no impregna la 'cultura' de los profesionales del hospital y causa en muchas ocasiones rechazo.

La falta de una definición clara de modelo de hospital universitario trae consigo diferentes consecuencias deletéreas:

- Dirección simultánea desde dos instituciones sobre los mismos recursos.

- Relaciones económicas y funcionales entre las dos instituciones muy mal definidas.

- Ausencia de interlocución eficaz entre instituciones.

- Centros docentes y departamentos universitarios ajenos a decisiones estratégicas en los hospitales, $\mathrm{y}$ viceversa. 
- Deterioro de la imagen de la universidad y sus profesionales.

- Generación de estructuras en el entorno del sistema de salud, preferentemente hospitalarias, para el desarrollo de funciones típicamente universitarias que compiten con la propia universidad.

- Ausencia de aprovechamiento de sinergias.

\section{Universidad de Ciencias de la Salud}

Para intentar solventar las dificultades del modelo español actual, Rozman et al, en un artículo de opinión reciente, apoyan la idea de la creación de una Universidad de Ciencias de la Salud a caballo entre el sistema sanitario y la universidad tradicional. Para ellos, las características de este modelo incluirían:

- Ente único dotado de personalidad jurídica propia.

- Naturaleza jurídica que permitiera promover la gestión clínica de los servicios y adoptar instrumentos de gestión empresarial.

- Composición mixta de sus órganos de gobierno, que integrara al tiempo la representación de la universidad y la institución sanitaria.

- Organización única, que permitiera superar la falta de correspondencia entre la estructura universitaria y la estructura de los hospitales.

- Desarrollo coetáneo de funciones docentes, de investigación y, en su caso, asistenciales, de todos los profesionales de esta universidad, que habrían de someterse a un régimen jurídico único.

- Organización flexible que hiciera posible ensayar nuevos modelos de educación médica.

\section{Hacia un nuevo modelo de hospital universitario}

Sin llegar al modelo de la Universidad de Ciencias de la Salud, y como paso previo e imprescindible para que un hospital universitario cumpla con las funciones que le corresponden, deberían adoptarse de forma inminente una serie de actuaciones que incluirían:

- Orientar planes estratégicos, objetivos e indicadores a la misión del hospital universitario.

- Revisar estructuras directivas y mandos inter- medios para que la triple misión esté adecuadamente representada.

- Estudio económico de los costes derivados de la función universitaria que incluya necesidades de personal docente e investigador.

- Modificación normativa universitaria y asistencial para que los trabajadores del hospital universitario participen en las funciones asistenciales y académicas.

- Creación de órganos de gestión conjunta de todos los recursos implicados.

- Desarrollo de unidades conjuntas técnico-administrativas para las tres funciones.

- Máximo nivel de coincidencia para los puesto de liderazgo de las estructuras académicas y asistenciales.

- Planificación, promoción y evaluación de los recursos humanos única y específica para los hospitales universitarios.

\section{Conclusiones}

El hospital universitario debe ser una estructura fundamental en la docencia de la medicina y otras ciencias de la salud, en la investigación e innovación biomédicas, en la transferencia conocimiento y en la formación continuada del Sistema Nacional de Salud.

El conjunto de dichas funciones, armonizado con una asistencia sanitaria de excelencia, es lo que define a un hospital universitario.

Para el cumplimiento de dichas funciones, el hospital universitario debe tener una estructura organizativa específica, que armonice y haga compatible su carácter propio.

Es necesario establecer procedimientos conjuntos de gestión y control que superen las limitaciones estructurales actuales de una dirección simultánea, desde diferentes instituciones, de las mismas funciones y recursos.

\section{Bibliografía}

1. Rozman C, Lafarga-Traver JL. La Universidad de Ciencias de la Salud: aspectos legales de una nueva opción. Med Clin (Barc) 2005; 124: 499-503.

2. Generalitat Valenciana. Hacia un nuevo modelo de hospital universitario. Valencia: Generalitat Valenciana; 2008. 